\title{
Swertz, Christian
}

\section{Bildungstechnologische Medienpädagogik}

formal überarbeitete Version der Originalveröffentlichung in:

formally revised edition of the original source in:

Sander, Uwe [Hrsg.]; Gross, Friederike von [Hrsg.]; Hugger, Kai [Hrsg.]: Handbuch Medienpädagogik.

Wiesbaden : VS, Verl. für Sozialwiss. 2007, S. 66-74

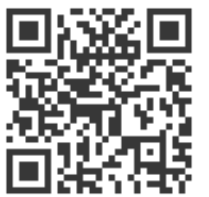

Bitte verwenden Sie in der Quellenangabe folgende URN oder DOI /

Please use the following URN or DOI for reference:

urn:nbn:de:0111-opus-73456

10.25656/01:7345

https://nbn-resolving.org/urn:nbn:de:0111-opus-73456

https://doi.org/10.25656/01:7345

\section{Nutzungsbedingungen}

Gewährt wird ein nicht exklusives, nicht übertragbares, persönliches und beschränktes Recht auf Nutzung dieses Dokuments. Dieses Dokument ist ausschließlich für den persönlichen, nicht-kommerziellen Gebrauch bestimmt. Die Nutzung stellt keine Übertragung des Eigentumsrechts an diesem Dokument dar und gilt vorbehaltlich der folgenden Einschränkungen Auf sämtlichen Kopien dieses Dokuments müssen alle Urheberrechtshinweise und sonstigen Hinweise auf gesetzlichen Schutz beibehalten werden. Sie dürfen dieses Dokument nicht in irgendeiner Weise abändern, noch dürfen Sie dieses Dokument für öffentliche oder kommerzielle Zwecke vervielfältigen, öffentlich ausstellen, aufführen, vertreiben oder anderweitig nutzen.

Mit der Verwendung dieses Dokuments erkennen Sie die Nutzungsbedingungen an.

\section{Terms of use}

We grant a non-exclusive, non-transferable, individual and limited right to using this document

This document is solely intended for your personal, non-commercial use. Use of this document does not include any transfer of property rights and it is conditional to the following limitations: All of the copies of this documents must retain all copyright information and other information regarding legal protection. You are not allowed to alter this document in any way, to copy it for public or commercial purposes, to exhibit the document in public, to perform, distribute or otherwise use the document in public.

By using this particular document, you accept the above-stated conditions of use.

\section{Kontakt / Contact:}

\section{peDOCS}

DIPF | Leibniz-Institut für Bildungsforschung und Bildungsinformation Informationszentrum (IZ) Bildung

E-Mail: pedocs@dipf.de

Internet: www.pedocs.de

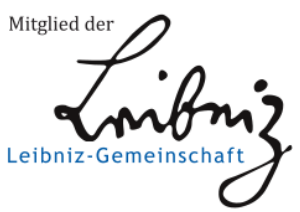




\section{Bildungstechnologische Medienpädagogik}

von Christian Swertz

\section{Einleitung}

Gegenstand der Bildungstechnologischen Medienpädagogik ist das pädagogische Verständnis von und die Aufklärung über Technologien und Techniken, die zur Verständigung in Bildungsprozessen verwendet werden. Technologien sind die Ideen oder Konzepte, die den zur Verständigung verwendeten Gegenständen zu Grunde liegen. Mit Technik wird der Gegenstand bezeichnet, der als Mittel der Verständigung verwendet wird. Beispiele für die Differenz zwischen Technologie und Technik sind die Technologie der Quadraturamplitudenmodulation, die in der Technik des analogen Farbfernsehers verwendet wird, oder die Technologie der Von-Neumann-Architektur, die in der Technik der meisten heute verwendeten Computer realisiert wird.

Während das Farbfernsehen vielen Menschen bekannt ist und sie in der Lage sind, diese Technik zu verwenden, ist die Technologie der Quadraturamplitudenmodulation meist nur ausgebildeten Fernsehtechnikerinnen und Fernsehtechnikern bekannt. Viele Menschen verwenden die Technik, ohne die Technologie zu kennen oder zu verstehen. Dasselbe gilt für die Von-Neumann-Architektur, die nur wenigen Nutzerinnen und Nutzern von Computern bekannt ist. Die meisten Menschen verwenden Computertechnik, ohne die Technologie zu kennen. Wenn eine Technik ohne Kenntnis der Technologie verwendet wird, dann wird der in der Technik ausgedrückte Verständigungsanspruch der Technologie unbewusst wirksam.

Eine Verwendung von Technik ohne Kenntnis der Technologie ist unproblematisch, wenn die unbewusste Verwendung einer Technik beabsichtigt ist. Die unbewusste Verwendung von Technik wird gelegentlich in politischen Forderungen an pädagogisches Handeln vertreten, in denen oft auch die wirtschaftliche Konkurrenzfähigkeit in den Mittelpunkt gestellt wird (z.B. CDU: 1998). Mit dieser Position wird die Gewöhnung von Menschen an Technik fokussiert. Eine solche Position ist aus Sicht der Pädagogik legitim, insofern pädagogisches Handeln immer an eine Alltagswelt von Menschen gebunden bleibt. Wenn die Alltagswelt der Menschen durch ein bestimmtes wirtschaftliches System gekennzeichnet ist, dann ist dies in pädagogischem Handeln zumindest auch zu berücksichtigen. Ausschließlich eine solche Begründung für pädagogisches Handeln zu verwenden greift aber zu kurz, da pädagogisches Handeln nicht nur an wirtschaftlichen, politischen oder religiösen Interessen, sondern auch an der Möglichkeit einer Vernunft zu orientieren ist. Die Möglichkeit einer Vernunft fordert ihre Realisierung und damit die Aufklärung über Technik. Für die Aufklärung über Technik ist die Darstellung und Analyse der Technologie ein möglicher Zugang, durch den untersucht werden kann, inwiefern eine Technik als Mittel der Verständigung in Bildungsprozessen zur Bildung von Menschen beiträgt. Der durch das Verständnis von Technologie 
mögliche vernünftige Umgang mit Technik ist das Ziel einer kritisch gedachten Bildungstechnologischen Medienpädagogik.

Ein Ansatzpunkt für eine kritisch gedachte Bildungstechnologische Medienpädagogik ist die Dialektik von Technik und Technologie. Diese kritische Aufgabe fordert eine spezifische Methode, denn die Bestimmung des mit dem Wort Kritik bezeichneten Begriffs kann nicht unabhängig von Technik und Technologie erfolgen. Die Bestimmung eines Begriffs setzt die Möglichkeit der Verständigung voraus und kann insofern nur durch den Gebrauch von Techniken und Technologien erfolgen. Der in der verwendeten Technik ausgedrückte Verständigungsanspruch fließt damit in das Verständnis von Begriffen ein. Dieser Einfluss kann jedoch bedacht werden. Dieses Bedenken ist einerseits durch die verwendete Technik begrenzt, die Begrenzung kann aber gleichzeitig reflektiert werden. Für die Analyse des Verhältnisses von Technik und Technologie in der bildungstechnologischen Medienpädagogik ist also eine dialektische Methode erforderlich, in der Technik zur Verständigung verwendet wird und zugleich selbstreflexiv die damit implizierten Voraussetzungen expliziert werden.

Aus Sicht der mittels selbstreflexiver Methode arbeitenden Bildungstechnologischen Medienpädagogik ist klar, dass Erziehung nicht nur technisch betrieben und Bildung nicht nur technologisch verstanden werden kann. Würde Bildung nur technologisch betrieben, dann würde die Frage nach dem Sinn und der Funktion von Technik in Bildungsprozessen und damit die Möglichkeit der Selbstreflexion übergangen. Bildung unterliegt aber keinem technologischen Ursache-Wirkungs- Verhältnis, wie dies etwa bei der Abarbeitung von Algorithmen durch Computertechnik der Fall ist: Der Algorithmus als Ursache bestimmt eindeutig, welche Wirkungen im Computer ausgelöst werden. Der Computer kann sich, wie an der Technologie dieser Technik zu erkennen ist, nicht in ein Verhältnis zur Verständigung setzen (Swertz 2000: 182), was Menschen jederzeit möglich ist. Durch dieses in ein Verhältnis setzen kann Technik selbstreflexiv verstanden werden. Es geht in der Bildungstechnologischen Medienpädagogik also nicht nur um Datenübermittlung im Sinne von Shannon (1976), sondern um Verständigung zwischen Menschen, für die eine Datenübermittlung allerdings eine notwendigen Bedingung darstellt. Mit der vorgeschlagenen dialektischen Methode wird dieser Prozess verstanden und nicht nur technisch erklärt. Im Verstehen des Prozesses ist zu reflektieren, dass Verstehen Verständigung und damit den Gebrauch von Technik und Technologien voraussetzt.

\section{Schrift}

Neben der Relation zwischen Technik und Technologie bestehen Relationen zwischen den zur Verständigung verwendeten Techniken und ihren Technologien. Die Relationen zwischen Techniken eröffnen einen Reflexionsraum, der es ermöglicht, die Relation zwischen einer Technik und ihrer Technologie in einer anderen Technik zu spiegeln und so eine Distanz aufzubauen, in der die Relation zum Gegenstand wird. Dadurch werden die Relationen zwischen Techniken zum Anlass 
der Reflexion. Ob Reflexion dabei als Ziel oder Reflexivität als Möglichkeit (vgl. Meder 2006: 11) in den Mittelpunkt von Bildung gestellt wird, kann hier außer Acht gelassen werden; entscheidend ist, dass die Relationen zwischen Techniken ein bildendes Moment enthalten.

Inwiefern dieses Moment zum Zeitpunkt des Aufkommens der gesprochenen Sprache vor vermutlich etwa 400.000 Jahren (vgl. Kuckenberg 2004) relevant ist, kann nicht rekonstruiert werden. Die Beobachtung von Tieren legt aber nahe, dass die Verständigung mittels Gesten zum Zeitpunkt der Entstehung von Sprache ebenfalls möglich ist, so dass von vornherein eine Relation zwischen Techniken der Verständigung besteht. Mit dem Aufkommen von Höhlenzeichnungen im Südwesten Europas vor ca. 30.000 Jahren stehen dann drei Techniken zur Verfügung, die mit dem Aufkommen der Schrift um 3.000 v. Chr. (vgl. ebd.) um eine vierte Technik erweitert werden.

Eines der frühesten für den europäischen Kulturraum relevanten Dokumente, in denen die Relation der zur Verständigung verwendeten Techniken zum Gegenstand gemacht wird, ist der Mythos von Theut, in dem der Götterbote Theut dem König Thamus aus Theben die Kunst der Schrift mit den Worten anpreist: "Dieser Lehrgegenstand, o König, wird die Ägypter weiser und gedächtnisfester machen; denn als Mittel für Gedächtnis und Weisheit ist er erfunden worden" (Phaidros 274 e). Thamus antwortet: "O Du Meister der Kunstfertigkeit, Theut: der eine ist imstande die Künste hervorzubringen, ein anderer, zu beurteilen in welchem Verhältnis Schaden und Nutzen sich verteilen werden für die Leute, die sie brauchen sollen. Auch du hast jetzt, als Vater der Schrift, aus Voreingenommenheit das Gegenteil von dem angegeben, was sie vermag. Denn diese Kunst wird Vergessenheit schaffen in den Seelen derer, die sie erlernen, aus Achtlosigkeit gegen das Gedächtnis, da die Leute im Vertrauen auf das Schriftstück von außen sich werden erinnern lassen durch fremde Zeichen, nicht von innen heraus durch Selbstbesinnen" (Phaidron 275 a-b).

Die Figur des Thamus, die Platon einer Erzählung des Sokrates entnommen hat, der sie wiederum in recht freier (jedenfalls nicht wahrer) Interpretation einer ägyptischen Erzählung verwendet hat (Apelt 1988: 142), urteilt zunächst in der Form des Dialogs über die Schrift. Allerdings handelt es sich bei dem Dialog um eine Schrift, und nicht um eine Rede. Es wird lediglich die rhetorische Form des Dialogs genutzt und in der Schriftsprache verwendet. Damit wird die Form des mündlichen Dialogs in der Schriftsprache reflektiert. Durch diese Reflexion wird auf das Verhältnis von Schrift und gesprochener Sprache aufmerksam gemacht. Unter Berücksichtigung dieses Spiels über und mit den Verständigungstechniken fällt auf, dass im Dialog zwar Vorteile der gesprochenen Sprache behauptet, gleichzeitig aber die Vorteile der Schrift demonstriert werden. Denn die vom Autor offenbar beabsichtigte Überlieferung wäre in mündlicher Form überhaupt nicht möglich gewesen.

Und auch die in der weiteren Argumentation von Platon thematisierte Authentifizierung von Geltung, die in der Schrift mangels Rücksprache nicht in der gleichen Form stattfindet wie beim Gebrauch der gesprochenen Sprache, ist im Mythos von Theut nicht so eindeutig als Nachteil der Schrift ausgewiesen. Denn dass auch Sprache zur Indoktrination verwendet werden kann, wird im 
Dialog durchaus reflektiert (Mitgutsch 2007: 101ff.).

Aus dieser Sicht erscheint das Argument des Thamus, die Stärkung des seelischen Vorganges des Erinnerns durch die Schrift sei von Theut nur behauptet, während sie in Wahrheit nur ein Mittel sei um sich zurückzuerinnern an das, was man vorher selbst verstanden und im Gedächtnis hat, als ein rhetorisch geschicktes Spiel. Die schriftliche Mitteilung des Argumentes macht deutlich, dass die Schrift durchaus selbstkritische Erkenntnis ermöglicht. Mit der Kritik an der Sprache im Dialog von Theut wird so verstanden nicht die Schrift kritisiert, sondern auf die in der Technologie liegenden Regeln der Verständigung hingewiesen, d.h. die Technologie analyisert.

Die Technologie des Verständigungsspiels wird durch die Relationierung der Techniken reflektiert. Das wird von Platon durch das Spiel mit der Form der Rede in der Schrift erreicht. Auf diesem Weg wird unter anderem reflektiert, welche impliziten Regeln eine Technik mit sich bringt. Dieser Aspekt ist für die Bildungstechnologische Medienpädagogik relevant, wenn Medienkritik als Bildungsziel verfolgt werden soll. Die medienpädagogische Medienkritik stellt meist die Inhalte, also die Bedeutung der an Technik gebundenen Zeichen, in den Mittelpunkt (Baacke 1996). Bleibt es bei der Kritik des Inhalts wird zum einen übersehen, dass nicht nur der Inhalt, sondern auch die zur Verständigung verwendete Technik kritisch reflektiert werden kann, zum anderen wird übersehen, dass eine Emanzipation von mittels Technik kommunizierten Machtansprüchen durch den Umstand begrenzt ist, dass auch der Emanzipationsanspruch mittels Technik mitgeteilt und dabei an die Struktur der verwendeten Technik angepasst werden muss. Dieses in der Bildungstechnologischen Medienpädagogik untersuchte Moment ergänzt daher die medienpädagogische Aufgabe der Medienkritik.

Im Mythos vom Theut wird die Reflexion zwischen zur Verständigung verwendeten Techniken sichtbar. Dass mit dem Spiel zwischen Technologien und Techniken nicht nur die Frage nach Wahrheit und Öffentlichkeit, sondern auch der Erziehung gestellt ist, zeigt die Reflexion des Buchdrucks bei Comenius.

\section{Druck}

Ein wesentlicher Anlass zur Reflexion von Verständigungstechniken, die in Bildungsprozessen eingesetzt werden, ist die Verbreitung des Buchdrucks im Anschluss an die erfolgreiche Anwendung von Matritzen zur Letternproduktion durch Gutenberg um 1450. Diese Erfindung hat nicht zuletzt die Entstehung der Didaktik beeinflusst, die schon bei Comenius eine Mediendidaktik ist: In der zwischen 1627 und 1657 erschienenen Opera didacta omnia macht Comenius den Buchdruck geradezu zum Maßstab für den Unterricht in und die Organisation von Schulen.

Comenius hebt als Vorteile des Buchdrucks hervor, dass zum einen die gedruckten Exemplare eines Buches ,,sich ähnlicher sind als ein Ei dem anderen - ein hübscher und prächtiger Anblick“ (Comenius 1992: 213) und dass nicht nur gutes, starkes Papier, sondern ,,auch dünnes Papier, Linnen 
usw.“ (Comenius 1992: 214) bedruckt werden kann. Er fährt im Blick auf die Organisation von Schulen fort: „Ganz Ähnliches wird sich zeigen, wenn einmal alles, was zu [unserer] neuen allgemeinen Form des Unterrichts gehört, eingerichtet ist“"(ebd.). Nach Comenius wird durch die breite Verwendung von Büchern zum einen das Unterrichten verbessert, da Lehrer alles unterrichten können, wenn sie nur den Stoff im Buch vor sich haben, und zum anderen das Lernen verbessert, da „die Wissenschaften beinahe in derselben Weise dem Verstande eingeschrieben [werden C.S.], wie sie äußerlich auf das Papier aufgedruckt werden.“(ebd.). Im weiteren entwickelt Comenius seine Vorstellung von Schule in Analogie zu den Materialien (Papier, Typen, Druckerschwärze, Presse) und Arbeitsgängen (Papierherstellung, Satz, Farbauftrag, Korrektur, Druck, Trocknung) des Buchdrucks. Als Bezeichnung für sein Verfahren schlägt er in Anlehnung an die Typographie den Ausdruck Didachographie vor. Zentrales Mittel der Didachographie sind Bücher: „Daher steht und fällt die ganze Sache mit der Beschaffung panmethodischer Bücher“ (Comenius 1992: 222).

Comenius macht die Technologie des Buchdrucks zum Maßstab für Erziehung, Unterricht und Lernen. Die Schule soll in Analogie zur Technik des Buchdrucks organisiert werden, und in der Schule sollen vor allem Bücher als Lernmaterialien verwendet werden - deren Inhalt sich dann in die Lernenden einschreibt. Diese Analogie ist nicht neu: Die Vorstellung, dass das Gehirn ebenso bedruckt wird wie das Papier, findet ihr historisches Vorbild in der tabula rasa bei Demkokrit, auf die sich Comenius durch seine Seneca - Rezeption bezieht (Comenius 1992: 35). Die häufig in der späteren Formulierung von Locke rezipierte unbeschriebene Wachstafel, in die durch einen Griffel Zeichen geprägt werden, wird zur Analogie der Bildung des Menschen, der bei Geburt unbeschrieben, aber zur Aufnahme der Schriftzeichen fähig gedacht wird (Benner/Brüggen 2001: 178).

Von Comenius wird damit die Drucktechnik als Metapher zum Verständnis des menschlichen Denkens herangezogen. Wenn auf diese Weise Techniken zum Verständnis des menschlichens Denkens verwendet werden, dann liegt ein abduktiver Schluss (Peirce 1976) vor. Die Funktion der bekannten Regel kommt in diesem Schluss der Technik zu, das Unbekannte, auf das geschlossen wird, ist der Mensch. Der Mensch ist das Unbekannte, das Ungeschriebene oder sogar Unschreibbare, und damit gefährlich, bedrohlich und also zu disziplinieren; die Technik ist das bekannte und damit das Gute. Mit der Vermutung, dass der Mensch wie eine Wachstafel oder ein zu druckendes Buch zu verstehen ist, erscheint es dann nicht mehr als rätselhaft, sondern als selbstverständlich, dass der Mensch ist, wie er ist. Die Technologie des Buchdrucks wird so nicht nur zur Verständigung zwischen Menschen, sondern auch zum Verständnis des Menschen und damit zum Selbstverständnis verwendet.

Dieses Moment rückt neben der Technik als Mittel der Verständigung Technologie als Mittel des Verstehens des Menschen in den Blick der Bildungstechnologischen Medienpädagogik. Es geht dabei nicht nur darum, den Menschen durch eine Analogie zu einer Technologie zu verstehen, sondern um die Frage, inwiefern es gerade die Technologie des in der Analogie herangezogenen Mediums ist, die ihrerseits bestimmte Vorstellungen vom Menschen prägt.

Aus Sicht der von Comenius vertretenen Pansophie ist die Maschine ebenso wie der Mensch 
Ausdruck des lebendigen Gottes (Schaller 1997). Es geht Comenius nicht um eine Unterordnung des Menschen unter die Technik, sondern um den Nutzen der Technik zu Ehren Gottes, zum Wohl der Menschen und zur Weltverbesserung. In der Technik tritt Gott als Retter der Welt in Erscheinung (Schaller 1997: 40). Es geht Comenius also nicht darum, Effizienzsteigerung in ökonomischem Interesse $\mathrm{zu}$ optimieren und $\mathrm{zu}$ forcieren, sondern darum, durch den natürlichen und also vernünftigen Gebrauch von Technik in der Erziehung zum Wohle des ganzen Menschen und der Menschheit beizutragen. Daher stellt Comenius auch den Gedanken des Gleichen in den Mittelpunkt. Es geht Comenius darum, „daß in den Schulen alle alles gelehrt werden müssen“ (Comenius 1992: 54).

Alles meint damit kein letztlich vollständiges Wissen, sondern bezeichnet wesentliche Grundlagen. Durch die Beschränkung auf Grundlagen, also eine Auswahl von Wissen, berücksichtigt Comenius die Reproduzierbarkeit von Wissen durch den Buchdruck. Die Vervielfältigung macht es angesichts der von Comenius als Ausgangspunkt gesetzten Begrenztheit des menschlichen Lebens unmöglich, alles zu lesen. Daher ist eine Auswahl nötig. Über diese Auswahl sollen jedoch alle gleichermaßen belehrt werden (Comenius 1992: 55). Dass alle Menschen das Gleiche lernen ist jedoch nur unter der Bedingung des Buchdrucks möglich, da erst die immer gleich gedruckten Bücher es überhaupt denkbar machen, alle Menschen gleich zu lehren.

Comenius erwartet durch den Buchdruck eine, modern gesprochen, eindrucksvolle Effizienzsteigerung des Unterrichts: Ein Lehrer soll genügen, um 100 Schülerinnen und Schülern durch vier Stunden Unterricht am Tag alles beizubringen (Comenius 1992: 64). Das soll durch einen Wechsel der Rolle des Lehrers hin zu einer Aufgabe erreicht werden, die heute als Moderation von kooperativen lebenslangen Lernprozessen zu beschreiben wäre (Comenius 1992: 71f., 84f.). Eine wesentliche Aufgabe des Lehrers wird die Zucht, also die Moderation des Lernprozesses durch die Disziplinierung der Schülerinnen und Schüler. Disziplinierung soll weniger durch Strafen erreicht werde, als durch die Kontrolle des Lesens: Alle Schülerinnen und Schüler sollen mit dem gleichen Buch lernen (Comenius 1992: 129f.). Da dies unter der Bedingung der Handschrift unmöglich ist, reflektiert die von Comenius vorgeschlagene Form der Disziplinierung die Technik des Buchdrucks, die so zur Disziplinierung des Denkens verwendet wird.

Gelernt werden soll nicht nur aus Büchern - die Buchdrucktechnik wird von Comenius nicht absolut gesetzt, sondern die Kenntnis der Dinge selbst ebenso betont wie die Notwendigkeit, über Gelesenes zu sprechen (Comenius 1992: 112, 123f.). Comenius entwirft also eine Struktur, die heute als multimodale Lernumgebung gelten würde. Die Unterrichtsgespräche sollen Unterrichtshandbüchern folgen, so dass auch die Lehrenden mittels Buchdruck diszipliniert werden. Diese Disziplinierung erfolgt bei Comenius zwar nicht im wirtschaftlichen Interesse, sondern im Interesse der Teilhabe am lebendigen Gott, ist aber dennoch eine Disziplinierung. Die Disziplinierung zum pansophischen Frieden für alle Menschen ist dabei aufs engste verbunden mit der durch den Buchdruck aufgekommenen Möglichkeit, allen Menschen das Gleiche zu unterrichten.

Zwar wird bei Comenius die Technik zum Maßstab von Schule, die Technologie wird aber noch nicht systematisch reflektiert. Möglicherweise entgeht Comenius deswegen, dass die Trennung, die 
sein Zeitgenosse und Kontrahent Descartes mit der starken Unterscheidung von res cogitans und res extensae machte, viel besser zum zergliedernden Wesen des Buchdrucks passt als der pansophische Gedanke. Mit der descartschen Trennung rückt die Gestaltung von Welt mittels Technik in den Focus; Gestaltung von statt Einfügen in Technologie wird zum entscheidenden Thema.

\section{Lehr- Lernmaschinen}

Dieser Gedanke wird bei Thorndike deutlich sichtbar: „If, by a miracle of mechanical ingenuity, a book could be so arranged that only to him who had done what was directed on page one would page two become visible, and so on, much that now requires personal instruction could be managed by print“" (Thorndike 1912: 165). Thorndike schlägt vor, die Technik des Buches so zu verändern, dass der Lernprozess genauer kontrolliert werden kann. Er möchte die Absicht der Kontrolle über den Lernprozess in Technik ausdrücken und schlägt dafür eine Veränderung der Technik des Buchdrucks vor. Die Buchseiten sollen nicht mehr vom Lernenden in beliebiger Reihenfolge gelesen werden können, sondern (ähnlich wie bei Comenius) in einer vorher vom Lehrenden festgelegten Reihenfolge. Dabei wird hier zwar noch das eher Comenius entsprechende Wunder genannt, es war aber klar, dass eine entsprechende Entwicklung eher von der Descartes folgenden menschlichen Erfindungsmacht erwartet wurde.

Thorndike forumliert dazu drei Bedinungen für Lernen: Bereitschaft, Übung und Auswirkung (Thorndike 1911). Zunächst wird die Bereitschaft etwas zu Lernen um einen angenehmen Zustand zu erreichen angenommen. Das Gelernte muss dann geübt werden, wobei eine Verhaltensweise nach Thorndike eher wiederholt wird, wenn sie zu positiven Konsequenzen führt.

Thorndike rückt zwar den Gedanken in den Mittelpunkt, dass ein Buch mehr leisten kann als nur Denkergebnisse oder Probleme zu präsentieren (Thorndike 1912: 165f.). Die von ihm entworfene Technologie war jedoch mit dem Buchdruck alleine nicht zu realisieren, und Thorndike selbst sah zwar, dass er damit das technische Problem aufgeworfen hatte, das heute als Interaktivität bezeichnet wird, hat selbst aber die Technik nicht realisiert.

Diese Realisierung wird im Anschluss an Thorndike von Pressey vorgenommen. Pressey war mit dem Problem konfrontiert, dass er häufig Wissen zu vermitteln hatte, bei dem es vor allem darauf ankam, das Gelernte genau so, wie es vermittelt wurde, wiederzugeben. Diese sich wiederholende Lehre des immer gleichen Materials wollte er lieber Maschinen überlassen, um so die Lehrenden von langweiliger Routinearbeit zu entlasten, und ,[...] make her free for those inspirational and thought-stimulating activities which are, presumably, the real function of the teacher" (Pressey 1926: 374). Es geht Pressey also keineswegs darum, Lehrende durch Technik zu ersetzen, sondern darum, die Teile des Bildungsprozesses, die ohne die Anwesenheit von Lehrenden vollzogen werden können (also einfache Formen des Lernens), durch Technik zu realisieren. Bildung soll keineswegs gänzlich mechanisiert und Lehrende durch Lernmaschinen ersetzt werden. Vielmehr geht es Pressey darum, für die deutlich begrenzten Aspekte des Bildungsprozesses eine Verständigung mittels 
Lehrmaschinen zu erreichen, für die das möglich ist.

Pressey (1926) montiert dazu eine Maschine ähnlich einer Schreibmaschine. Vor eine Walze baut er ein Fenster, durch das eine Frage mit vier Antworten sichtbar ist. Durch die Walze kann das Papier wie bei der Schreibmaschine weitertransportiert werden. Auf die Seite der Maschine montiert er vier Tasten, mit denen Lernende die Antwort auswählen, die sie für richtig halten. Dabei kann die Maschine zwischen Lehr- und Prüfungsmodus umgeschaltet werden: Im Lehrmodus wird die nächste Frage nur nach einer richtigen Antwort angezeigt. Die richtige Antwort kann durch Ausprobieren gefunden werden. Das Gerät liefert eine sofortige Rückmeldung, indem es zeigt, ob die Antwort richtig ist oder nicht.

Damit wird die von Thorndike formulierte Technologie des Effekts technisch realisiert. Zugleich rückt die Interaktivität mit der Maschine in den Blick: eine mechanische Reaktion teilt mit, ob eine Antwort richtig ist oder nicht. In einer weiteren Version (Pressey 1927) musste die richtige Antwort zweimal gegeben werden, um das Gesetz der Übung zu berücksichtigen. Pressey realisiert so die von Thorndike formulierten drei Bedingungen des Lernens in einer Technik. Weite Verbreitung fanden seine Ideen jedoch nicht, da durch die große Depression und den Zweiten Weltkrieg Lernprobleme aus dem Zentrum das gesellschaftlichen Interesses rückten.

Presseys Ideen wurden später von Skinner aufgegriffen. Dabei wurde vor allem das Bild der Black Box wirksam, durch die der Mensch als programmierbare Maschine erschien. Dieses Bild wurde von Skinner (1953) selbst jedoch explizit abgelehnt, da, so Skinner, Aussagen über Lernvorgänge nur von sich selbst Beobachtenden getroffen werden können. Er betrachtet den Menschen als ganzes Individuum, das auch aufgrund der individuellen Lebenserfahrungen und der Phylogenese lernt. An dieser Stelle ist ein kurzer Rekurs zum hier exponierten Verständnis Bildungstechnologischer Medienpädagogik erforderlich. Bildungstechnologische Medienpädagogik wird von Schorb (1998) mit der Verbindung von Lehrmaschinen und operandem Konditionieren gleichgesetzt. Unterricht solle aus Sicht Bildungstechnologischer Medinepädagogik nur noch mit Lehrmaschinen erfolgen, die dann die Lehrenden ersetzen. Dass Lehrmaschinen so nicht gedacht worden sind und Bildungstechnologische Medienpädagogik aus systematischen Gründen weiter gefasst werden muss, wurde bereits deutlich. Auch ein einfaches Ablehnen der Bedingung der Auswirkung (Thorndike) bzw. des operanden Konditionierens (Skinner) mit dem Verweis darauf, dass das operande Konditionieren der Bildung von Menschen nicht gerecht wird, greift zu kurz. Zwar ist klar, dass operandes Konditionieren alleine für Bildung nicht ausreicht. Das gilt jedoch für jede andere Unterrichtsmethode auch, und das schlechthin nichts durch operandes Konditionieren gelernt wird und keinerlei Bildung angeregt werden kann ist kaum plausibel zu machen - die überzeugende Nutzung des operanden Konditionierens in der Angsttherapie ist dabei nur ein aktuelles Beispiel (vgl. Margraf 2000). Daher gilt es zu fragen, wann die Technologie der Lehrmaschinen sinnvoll ist und wann nicht.

Die Konstruktion der Lernmaschinen durch Pressey macht jedenfalls deutlich: Technologie realisiert 
sich nicht selbst als Technik. Es ist stets ein Mensch, der eine Technologie in einer Technik realisiert, und durch diese Realisierung eine Verständigungsabsicht in der Technik ausdrückt. Das kennzeichnet eine Aufgabe der Bildungstechnologischen Medienpädagogik. Es geht darum, Technologie dem Bildungsgedanken gemäß zu entwerfen und als Technik zu realisieren. Für die Gestaltung von Bildungsprozessen ist es dabei wichtig, die Absichten, die in der Technik ausgedrückt sind, vernünftig zu gestalten.

Aus dieser Sicht sind Lernmaschinen und viele daran anschließende Ideen durchaus ein akzeptables Mittel für den Unterricht. Dass Lernmaschinen alleine nicht geeignet sind um Bildung zu erreichen ist klar - so kann z.B. komplexes Wissen nicht mittels Lernmaschinen vermittelt werden (Kulik/Kulik/Cohen 1980). Dass aber die mit Lernmaschinen mögliche Verständigung als ein Element in Bildungsprozessen durchaus sinnvoll sein kann ist schon dann offensichtlich, wenn Bildung nicht ohne bloße Kenntnis von etwas möglich ist. Für die Vermittlung von Faktenwissen haben sich Lernmaschinen (oder später die programmierte Unterweisung) jedoch durchaus als sinnvoll erwiesen.

Bei Thorndike, Pressey und Skinner findet sich ebenso wie bei Comenius der Gedanke, dass durch eine geeignete Technik Lernen in Erziehung und Unterricht verbessert werden kann, und auch die Idee der festen und optimalen Reihenfolge wird fortgeschrieben. Dabei wird die Verwendung der Technik allerdings nicht mehr auf einen pansophischen Gedanken bezogen. Der einzelne Mensch rückt in den Mittelpunkt der Bemühungen.

\section{Computer}

Der Aufgabe von Bildungstechnologischer Medienpädagogik, Technik für die Verwendung on Bildungsprozessen zu gestalten, korrespondiert die kritische Analyse des bildenden Gehalts von Technologien. Aktuell ist dabei vor allem die Computertechnologie relevant, die für viele Menschen zum zentralen Medium geworden ist. Eine kritische Analyse des bildenden Gehalts der Computertechnologie, der ich in diesem Abschnitt folge, hat Meder (1998) vorgelegt.

Meder zeigt zunächst, dass Computertechnologie zur pädagogischen Aufgabe wird, weil es sich um eine Kulturtechnik handelt. Eine Technik gilt als Kulturtechnik, wenn sie das ganze gesellschaftliche Leben auf allen Ebenen menschlicher Aktivitäten durchdringt und zugleich ein gesellschaftliches Problem betrifft, dessen Lösung als ein Wert an sich angesehen wird. Computertechnologie durchdringt das gesellschaftliche Leben durch die bloße Verbreitung und als abstrakter Problemlösungsautomat. Letzteres ist relevant, da nach Meder in der Moderne Welt nur noch als Problem wahrgenommen werden kann, und daher überall Computer für Problemlösungen verwendet werden können. Die Lösung der Probleme wird als Wert an sich angesehen, wenn die Lösung tradiert wird. Die Tradierung geschieht in der Moderne im Modus des Widerstreits um Geltung. Computertechnologie ermöglicht diesen Widerstreit um Geltung, weil sie als abstrakter Problemlösungsautomat mit jeglichen digitalisierbaren Inhalten gefüllt werden kann. 
Meder analysiert die Verwendung dieser Kulturtechnik in Bildungsprozessen. Der Bildungsprozess ist für ihn zunächst die Herstellung eines individuellen Verhältnisses zur Welt, zur Gesellschaft und zu sich selbst. Die drei Verhältnisse sind sprachlich vermittelt. Mittels Computertechnik werden diese Verhältnisse auf spezifische Weise gebildet. Das Spezifische der Computertechnologie ist:

1. Sprachliches Problemlösen wird durch die Erfindung und Erprobung von geeigneten Sprachen in universellen Turingautomaten ermöglicht. Computertechnologie macht es dabei erforderlich, nicht nur die Sprache, sondern auch die Konstruktionsprinzipien der sprachlichen Verhältnisse zu reflektieren. In der Computertechnik wird so eine doppelte Reflexivität ausgedrückt.

2. Diese Struktur der doppelt reflexiven sprachlichen Problemlösungen kennzeichnet auch das mit Computern mögliche Probehandeln. Probehandeln, das grundsätzlich bildungsrelevant ist, wird mit Computertechnik einfacher als mit anderen Medien und kann konkret sinnlich eingeübt werden.

3. Kommunikation als Moment aller drei Verhältnisse von Bildungsprozessen wird mit Computertechnik erweitert und beschleunigt. Die Kommunikation mit der Technik wird durch den Bildschirm als Oberfläche zu einem multimedialen Spiel mit Superzeichen.

4. Unser Verhältnis zu anderen wird ästhetisiert. Selbstdarstellung als Performanz eines kontingenten Entwurfs rückt in den Mittelpunkt des Bildungsgedankens. Diese Ästhetisierung findet sich auch in unserem Verhältnis zur Welt. Es geht in computervermittelten Bildungsprozessen um die beste mögliche Darstellung von Welt, nicht die Darstellung der besten Welt. Pluralität rückt in den Mittelpunkt, und es geht nicht mehr um Einheit, sondern um die feine Differenz. Der gebildete Mensch als Sprachspieler bewahrt in der feinen Differenz seine Eigenständigkeit auch gegenüber der Gesellschaft.

Schreibt Computertechnologie sich als Technik dem Menschen in Bildungsprozessen ein, dann wird der Gebildete zum Sprachspieler. Dabei steht im Mittelpunkt, dass Computer im Bildungsprozess das Reflexiv-werden von Sprache erfordern. Sprache als Mittel der Reflexion des Verhältnisses zur Welt wird damit als Mittel der Reflexion auf dieses Verhältnis verwendet, und damit doppelt reflexiv. Diese doppelte Reflexion kennzeichnet den Sprachspieler und daher auch den Ansatz dieses Aufsatzes.

Die Analyse von Meder zeigt, dass die Verwendung von Computertechnik in Bildungsprozessen dem Bildungsideal des Sprachspielers entspricht, und das Bildungsideal des Sprachspielers zugleich die Technologie der Computertechnik reflektiert. Hier wird zugleich deutlich, wie Pluralität als erkenntnistheoretische Prämisse die Möglichkeit der Erfindung von Sprachen als Moment der Computertechnologie reflektiert. Der Sprachspieler jedenfalls kennt sein doppelt reflexives Verhältnis zu sich selbst, zu anderen und zur Welt und beherrscht so das Spiel mit der Sprache zur Ausübung von Macht und Gewalt in der Informationsgesellschaft.

Zwar erscheint der Gedanke der gleichen Bildung für alle, wie Comenius ihn unter der Bedingung 
des vereinheitlichenden Buchdrucks formuliert hatte, unter der Bedingung der pluralisierenden Computertechnologie als nicht sinnvoll. Ebenso wie der Buchdruck einen Beitrag zur Bildung des Menschen leisten konnte ist dies jedoch auch mit Computern möglich. Wenn die Verwendung von Computern dabei auf den Gedanken des Gebildeten bezogen bleibt behält die pädagogische Verwendung von Computern ihre kritische Dimension. Die Kritik von gesellschaftlichen Anforderungen im Blick auf die pädagogische Aufgabe wird damit als zentrale Aufgabe Bildungstechnologischer Medienpädagogik realisiert.

\section{Medien}

Bildungstechnologie bezeichnet nicht Bildung als Technologie, sondern die Technologie der in Erziehung und Bildung verwendeten Techniken. Diese Techniken sind Medien. Um Medien handelt es sich immer dann, wenn Gegenstände von Menschen zum Zweck der Verständigung als Zeichen verwendet werden. In Medien als Gegenständen, die von Menschen als Zeichen verwendet werden, stehen Subjekt, Gegenstand und Zeichen in dialektischen Relationen zueinander. Daraus ergeben sich verschiedene Perspektiven:

(1) Wenn Medien artifizielle Gegenstände sind, kommt im Medium als Gegenstand eine Verständigungsabsicht zum Ausdruck. Medien werden also in der Rezeption als etwas verstanden. Dieser Aspekt steht im Mittelpunkt, wenn Medien in Bildungsprozessen gebraucht werden.

(2) Medien werden, wenn sie in der Relation zwischen Medien zum Gegenstand der Reflexion gemacht werden, als Zeichen verstanden. Dieser Aspekt steht im Mittelpunkt, wenn in Bildungsprozessen über Medien unterrichtet wird.

(3) Medien können gestaltet werden. Dieser die Mediendidaktik (vgl. Kerres in diesem Band) ergänzende Aspekt steht im Mittelpunkt, wenn Medien für Bildungsprozesse entworfen werden.

Diese Perspektiven zu bedenken und dabei zum einen die in Medien ausgedrückte Verständigungsabsicht im Interesse einer vernünftigen Verwendung von Medien und einer Aufklärung über Medien zu reflektieren und zum anderen Technologien zu entwerfen und in Technik zu realisieren ist die Aufgabe Bildungstechnologischer Medienpädagogik, in der die disziplinierende Gewöhnung an Technik und die Aufklärung über Technik aneinander gebunden bleiben.

Literatur

Apelt, Otto (1988): Platon. Menon. Oder über die Tugend. Leipzig: Felix Meiner Verlag. 
Baacke, Dieter (1996): Medienkompetenz als Netzwerk. In: medien praktisch 2. 1996. 4-10.

Benner, D./Brüggen, F. (2001): Bildsamkeit/Bildung. In: Benner, D.; Oelkers, J. (2001): 174-215.

Benner, D./Oelkers, J. (2001): Historisches Wörterbuch des Pädagogik. Weinheim und Basel: Beltz.

Borrelli, M./Ruhloff, J. (Hrsg.) (1998): Deutsche Gegenwartspädagogik Bd. III. Baltmannsweiler: Schneider Verlag.

CDU (1998): Zukunftsprogramm. Bonn.

Comenius, J. A. (1992): Große Didaktik. Stuttgart: Klett.

Kuckenburg, M. (2004): Wer sprach das erste Wort? Die Entstehung von Sprache und Schrift. Stuttgart: Theiss.

Kulik, J.A./Kulik, C./Cohen, P. (1980). Instructional Technology and College Teaching. In: Teaching of Psychology 7. 1980. 199-205.

Meder, N. (1998): Neue Technologien und Erziehung/Bildung. In: Borrelli, M./Ruhloff, J. (1998): 26-40.

Meder, N. (2006): Theorie der Medienbildung. In: Sesink, W.; Kerres, M.; Moser, H. (2006): 1-20.

Margraf, J. (2000): Lehrbuch der Verhaltenstherapie. Berlin: Springer.

Mitgutsch, Konstantin (2007): Indoktrination als Phantom. In: Schluß, Henning (Hrsg.):

Indoktrination und Erziehung - Einblicke in die Rückseite der Pädagogik. VS-Verlag.

Peirce, Charles Sanders (1976). Schriften zum Pragmatismus und Pragmatizismus. Frankfurt am Main: Suhrkamp.

Pressey, S. L. (1926): A simple apparatus which gives tests and scores - and teaches. School and Society. 23. 586. 373-376.

Pressey, S. L. (1927): A machine for automatic teaching of drill material. School and Society, 25. 645. 549-552.

Sesink, W.; Kerres, M.; Moser, H. (2006): Jahrbuch Medienpädagogik 6. Wiesbaden: VS - Verlag.

Schaller, Klaus (1997): Die Maschine als Demonstration des lebendigen Gottes: Johann Amos

Comenius im Umgang mit der Technik. Baltmannsweiler : Schneider-Verlag Hohengehren.

Schorb (1998): Stichwort: Medienpädagogik. In: Zeitschrift für Erziehungswissenschaft, 1, S. 7-22

Shannon, C. E. (1976): Die mathematische Theorie der Kommunikation. In: Shannon, C. E.; Weaver, W.: Mathematische Grundlagen der Informatiosnstheorie, S. 41-130. 
Skinner, B. F. (1953): Science and human behaviour. http://www.bfskinner.org/SHBtext.pdf (7.5.2007).

Skinner, B. F. (1958). Teaching machines. In: Science. 3330. 128. 969-977.

Swertz, C. (2000): Computer und Bildung. Bielefeld.

Thorndike, Edward L. (1911): Animal Intelligence. http://psychclassics.yorku.ca/Thorndike/Animal/ (7.5.2007). 